\title{
Activation of TRPA1 by Volatile Organic Chemicals Leading to Sensory Irritation
}

\author{
Jeanelle M. Martinez and Thomas E. Eling
}

National Institutes of Environmental Health Sciences, NIH, Research Triangle Park, NC, USA

\begin{abstract}
Many volatile organic chemicals (VOCs) have not been tested for sensory pulmonary irritation. Development of an in vitro non-animal sensory irritation assay that is suitable for a large number of chemicals is needed to replace the mouse assay. An adverse outcome pathway (AOP) is designed to provide a clear description of the biochemical and cellular processes leading to toxicological effects or an adverse outcome. The AOP for chemical sensory pulmonary irritation was developed according to the Organization for Economic Co-operation and Development guidance including the Bradford Hill criteria for weight of evidence to determine the confidence in the AOP. The proposed AOP is based on an in-depth review of the relevant scientific literature to identify the molecular initiating event (MIE) for respiratory irritation. The activation of TRPA 1 receptor (transient receptor potential cation channel, subfamily A, member 1 ) is the MIE leading to sensory irritation. A direct measure of TRPA 1 activation in vitro should identify chemical sensory irritants and provide an estimate of potency. Fibroblasts expressing TRPA 1 are used to determine TRPA 1 activation and irritant potency. We report a linear relationship between the in vivo $R_{50}$ and the in vitro $p E C_{50}$ values $(R=0.81)$ to support this hypothesis. We propose that this in vitro assay, after additional analysis and validation, could serve as a suitable candidate to replace the mouse sensory irritation assay.
\end{abstract}

\section{Introduction}

Inhalation exposure to gases and vapors frequently occurs in occupational and ambient environments. Each year new volatile organic chemicals (VOCs) are introduced into commercial use as solvents, vapors or gases and are subsequently incorporated into household consumer products and ultimately discharged into the environment. Many of these VOCs are not well characterized with respect to sensory irritation.

The term sensory irritation (Alarie, 1981) is frequently used to describe adverse health effects caused by compounds interacting with peripheral nerve fibers. The respiratory tract is richly innervated by trigeminal nerves and their nerve endings in the epithelial tissue of the nasal mucosa express transient receptor potential (TRP) ion channels (Fujitta et al., 2008; Bessac and Jordt, 2008; Inoue and Bryant, 2005; Bautista et al., 2006). Activation of TRP ion channels is involved in the detection of irritants pres- ent in the air. Exposure to chemicals by inhalation causes trigeminal chemoreception (pain, nasal pungency, eye irritation, decreases in respiration rate), which is dependent on the concentration and frequency of exposure.

TRPA1 (transient receptor potential ankyrin-repeat 1) and TRPV1 (transient receptor potential cation channel subfamily $\mathrm{V}$ member 1) are present in the nerve endings in the upper airways that mediate sensory irritation as part of a physiological response to make the subject aware of the presence of chemicals and initiate several defensive biological responses. A wide range of chemicals activates TRPA1, while acids and a limited number of chemicals activate TRPV1 (Bessac and Jordt, 2010). The activation of TRPA1 has been characterized as a gatekeeper of inflammation and is an essential endpoint in the regulation of exposure to VOCs (Bautista et al., 2013; Lehmann et al., 2016, 2017). TRPA1 is a regulator of neuropeptide release and neurogenic inflammation leading to increased mucus production,
Received November 1, 2018; Accepted March 29, 2019 ; Epub April 18, 2019; () The Authors, 2019.

ALTEX 36(4), 572-582. doi:10.14573/altex.1811012

Correspondence: Jeanelle M. Martinez, PhD

EASi/Aerotek LLC, providing services for Ethicon,

a Johnson \& Johnson medical device company

4545 Creek Rd, Cincinnati OH 45242, USA

(jmart139@its.jnj.com)

Thomas E. Eling, PhD

National Institutes of Environmental Health Sciences,

111 TW Alexander Dr. Research Triangle Park, NC 27709, USA

(teling6@gmail.com)
This is an Open Access article distributed under the terms of the Creative Commons Attribution 4.0 International license (http://creativecommons.org/licenses/by/4.0/) which permits unrestricted use, distribution and reproduction in any medium, provided the original work is appropriately cited. 
nasal obstruction, sneezing, and coughing (Belvisi et al., 2011; Bautista et al., 2013; Caceres et al., 2009).

Chemicals that are irritants can be identified and characterized based on the following: physical and chemical characteristics, the result of classical in vitro skin and eye corrosion irritation assays (Bos et al., 2002), and data obtained from in vivo sensory irritation tests (Alarie, 1966, 1981; Alarie et al., 1995). Acute inhalation injury by corrosive chemicals is overt and therefore easily quantified in animal studies. Corrosion is identified based on histological necropsy observations, including cell necrosis, inflammatory cell infiltration, and edema.

While physical and chemical characteristics are frequently predictive for corrosive chemicals, known sensory irritants have a wide range of physical and chemical characteristics that are, in general, not predictive for sensory irritation. There is only a single assay for the determination of sensory irritation, i.e., the classical in vivo sensory irritation test, which presents the result as the dose causing a $50 \%$ reduction in respiration rate or $\mathrm{RD}_{50}$ (Alarie, 1966, 1973, 1981; Alarie et al., 1995; Schaper, 1993). Many VOCs are classified as sensory irritants based on results from this assay. The $\mathrm{RD}_{50}$ has been proposed for developing guidance levels of $0.033 \times \mathrm{RD}_{50}$. However, the utility and validity of the $\mathrm{RD}_{50}$ assay for sensory irritation and the subsequent development of exposure limits have been questioned (Bos et al., 1991, 2002) as extrapolation of animal data to humans did not correlate with observed histopathological changes or corrosion of the respiratory tract. However, Kuwabara et al. (2007) and Nielsen and Wolkoff (2017) later pointed out that the RD 50 assay is designed to evaluate sensory irritation potential and not corrosive effects. Nielsen and Wolkoff (2017) propose a systematic approach for using animal data to set air quality guidelines that includes evaluation of the number of mice and the strain, exposure concentrations, exposure-response relationships, and the mode-of-action in mice and humans.

Improved or additional assays are needed to develop non-animal testing for sensory irritation. Ideally, the assay should be an in vitro system that can measure a reasonably large number of chemicals and is based on biochemical processes and chemical structure activity. A clear understanding of the biochemical and cellular mechanisms of receptor activation and signal transduction pathways for sensory irritation should point the way toward the development of non-animal assays for risk assessment of inhalable chemicals.

The Organization for Economic Co-operation and Development (OECD) developed a collaboration between the Joint Research Centre of the European Commission, the United States Environmental Protection Agency (US EPA), and the United States Army Engineer Research and Development Center, which resulted in the development of the Adverse Outcome Pathway Knowledge Base as a tool for risk assessment. An adverse outcome pathway (AOP) provides a clear description of the mechanisms underlying biochemical events and modes of action leading to adverse effects. Here, we report the development of an AOP for sensory irritation that provides guidance for designing in vitro assays for assessing and quantifying sensory irritation. This AOP (AOP \#196) is under development in the AOP-wiki, not open for comment, and can be found at https://aopwiki.org.

\section{Approach}

An AOP-wiki was developed based on Villeneuve et al. (2014) and the respective OECD guidelines (OECD, 2012a,b, 2018). All AOPs have a single starting point, i.e., the molecular initiating event (MIE), progress through several key events (KE) and culminate in a single endpoint or adverse outcome (AO), either in an individual or a population (OECD, 2012a,b, 2018). For VOC induced irritation, binding to sensory receptors was determined to be the MIE, and the AO is respiratory irritation. Identification of key biochemical and cellular events between the MIE and the AO was achieved by an in-depth, comprehensive survey of appropriate scientific literature, using PubMed and Google Scholar as the main resources along with reference tree searching.

\subsection{Relevant background information}

\subsubsection{Biochemical and cellular basis for sensory irritation}

Chemesthesis, i.e., sensations produced by chemical exposure, occurs through trigeminal nerve fiber endings present in the nasal cavity and airways and is mediated by a family of ion channel receptors located in the nerve endings.

The ASIC receptors are ubiquitously expressed in both the A and $\mathrm{C}$ fiber sensory neurons. Activation of these receptors plays a role in the pain response that occurs upon inhalation of chemicals that alter cellular acidity. ASIC receptors (acid-sensing ion channels) detect changes in acidity between pH 6 to 7. For example, inhalation of acetic acid activates ASIC receptors (Bessac and Jordt, 2010).

TRPV1 is a member of the TRP family ion channel receptors also located in the sensory fibers in the lining of the trachea, bronchi, and alveoli. TRPV1 is activated by a few chemicals and some irritants found in foods, e.g., chili peppers (capsaicin), black pepper, and garlic. TRPV1 is also activated by acidic conditions in the respiratory tract. Activation of TRPV1 causes respiratory tract irritation with sneezing, cough, increased mucus secretion, and pain. Activation of the TRPV1 receptor can desensitize the airway response to other irritants (Bessac et al., 2008; Bessac and Jordt, 2010).

TRPA1, which is present in the C-fiber nerve endings, is directly activated by VOCs and is essential for the irritant response (see 2.1.2). TRPA1 receptor reacts with or is activated by a wide range of natural and many environmental compounds, including dust, cigarette smoke, vapors, and common air pollutants. Electronic cigarettes can also produce potentially reactive aldehydes that may activate the TRPA1 receptor (Rowell and Tarran, 2015). Activation of the TRPA1 channel causes the release of pro-inflammatory cytokines at the site of interaction (Bessac and Jordt, 2010). The range of chemicals activating TRPA1 is very diverse, with the chemical reactivity of TRPA1 agonists being more important than their structure (Peterlin et al., 2007). TRPA1 receptors are also present in skin, suggesting that skin could be used as a surrogate for the respiratory tract in the in vitro investigation of sensory irritation by chemicals (Jordt et al., 2004).

TRPA1 and TRPV1 co-localize in the sensory nerve ending (Bessac and Jordt, 2008) and are expressed in the small diameter 
neurons in the trigeminal nerves located in the respiratory tract. The vagal jugular and vagal nodose ganglia project TRPA1-expressing C-fibers into the airways of the pulmonary system (Belvisi et al., 2011). TRPV1 is localized with substance P and NK1 receptors in trigeminal nerve endings and some evidence suggests that they interact with TRP receptors (Naono-Nakayama et al., 2010).

Upon activation of trigeminal neurons, the release of substance P (SP), a peptide that stimulates an inflammatory response, results in an increase in mucus secretion, plasma extravasation, and vasodilation. The nerve endings expressing neuropeptide CGRP (calcitonin gene-related peptide) in the respiratory tract are in the same regions as SP, and CGRP is frequently released with SP after stimulation (Russell et al., 2014). Nerve endings expressing CGRP are also located in the walls of small blood vessels and, after release from nerves, cause prolonged vasodilation. CGRP has two forms, $\alpha$ and $\beta$, with the expression of the $\alpha$-form abundant in the nerve fibers, respiratory tract mucosa, and pulmonary epithelium. Activation of the TRPA1, but not TRPV1, ion channel receptors in the airways can cause the release of CGRP (Kichko et al., 2015).

TRPA1 is expressed in many species with the activation by electrophiles conserved across species. Sensory detection of chemicals causes the following sensations: piquancy, tingling, prickling, irritation, stinging, burning, and pain, or may induce involuntary, autonomic, and motor reflexes (Bessac and Jordt, 2010). Also, the TRP ion channels are implicated in the cough reflex, a response observed upon inhalation of an irritant.

\subsubsection{TRPA 1 activation is essential for VOC induced irritation}

Activation of the TRPA1 channel appears to be the determinate for most of the response of the pulmonary system to inhaled irritants (Bessac et al., 2009; Belvisi et al., 2011; Caceres et al., 2009). Many known irritants, including isocyanates, cigarette smoke extracts, ozone, $\mathrm{H}_{2} \mathrm{O}_{2}$, and aldehydes, increase calcium flux in TRPA1-transfected HEK293 but not in control HEK293 cells. Calcium flux measured in isolated trigeminal neurons after incubation with an irritant chemical was significantly reduced in neurons isolated from TRPA1 deficient (TRPA1-/-) compared to wild-type mice (Taylor-Clark et al., 2009; Taylor-Clark and Undem, 2010). Furthermore, the irritation responses including cough, pain, and inflammation were absent or decreased after ex- posure of TRPA1-/- mice to irritants such as alkyl isothiocyanate, toluene diisocyanate, or formaldehyde (McNamara et al., 2007; Taylor-Clark et al., 2009). In contrast, TRPV1-/- mice exposed to acrolein did not respond with a decrease in respiration rate (Symanowicz et al., 2004). Therefore, the chemical activation of TRPA1 appears to be essential for the irritant response in the respiratory tract after exposure to pulmonary irritants (Belvisi et al., 2011).

\subsubsection{TRPA 1 and airway hyper-reactivity}

Hyper-activation and chronic activation of TRPA1 appear to contribute to chronic bronchitis, occupational asthma, and other inflammatory respiratory tract diseases associated with exposure to inhaled toxic agents. TRPA1 may also be important in allergic sensitivity because, as shown in experimental animals, the receptor modulates sensitivity to ovalbumin, an inducer of allergic asthma in experimental animal models (Bessac and Jordt, 2010). Activation of the irritation or nociceptive receptors can lead to increased sensitization to allergic stimuli (Bessac and Jordt, 2008, 2010).

The activation of TRPA1 stimulates the release of SP and CGRP, which play a role in the asthmatic and irritation symptoms observed after one-time and frequent exposure to inhaled irritants (Caceres et al., 2009). Exposure to high levels of TRPA1 agonists can induce reactive airway dysfunction syndrome (RADS), characterized by asthma-like symptoms (Brooks et al., 1985). Bessac and Jordt (2008) proposed that exposure to an irritant that activates TRPA1 may sensitize TRPA1 through inflammatory pathways, thereby establishing hypersensitivity to other reactive irritants.

TRPA1, but not TRPV1, appears to play an important role in allergic airway inflammation and hyper-reactivity associated with asthma. In the ovalbumin mouse model of asthma, TRPA1-/- mice, but not TRPV1-/-, had reduced mucus, fewer leukocytes in the bronchoalveolar lavage fluid, and lower amounts of inflammatory cytokines than wild-type mice. Exposure of mice to tear gas or TRPA1 agonist increased CGRP, SP, and NKA in wild-type mice while reduced levels were detected in the alveolar fluid of TRPA1-/- mice. Likewise, exposure of wildtype mice to ovalbumin increased levels of NKA while reduced levels were measured in TRPA1-/- mice. These results indicate that TRPA1 may also play a role in the development of asthma after allergen challenge (Caceres et al., 2009).

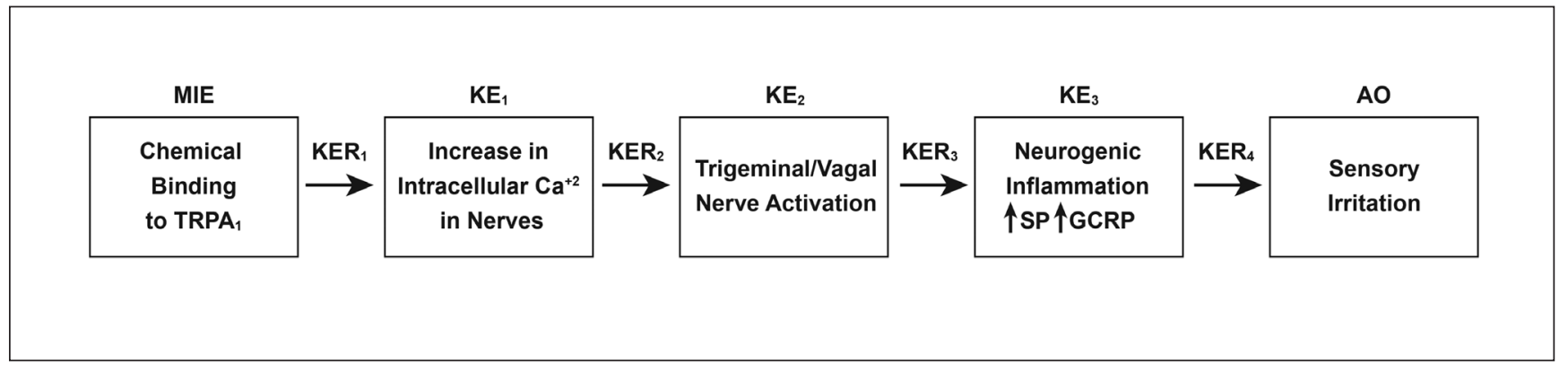

Fig. 1: The temporal sequence of biological events of the AOP (adverse outcome pathway)

MIE, molecular initiating event; KE, key events; KER, key events relationship; $A O$, adverse outcome 


\section{Sensory irritation AOP}

Based on the biochemical and cellular data on sensory irritation described above, we propose an AOP for sensory irritation as depicted in Figure 1.

\subsection{Molecular initiating event}

The binding of the irritant to the TRPA1 receptor is the MIE leading to an adverse outcome. Chemical irritants have highly diverse chemical structures but can be divided into two groups based on their chemical reactivity, i.e., reactive and non-reactive.

Irritants in the reactive group include endogenous inflammatory lipids, oxidants, and electrophilic agents that react with biomolecules including amino acid residues in proteins (Bessac and Jordt, 2010). Some members of this reactive group are isocyanates (tear gases), $\alpha, \beta$-unsaturated aldehydes, heavy metals, and peroxides. They are very potent irritants that covalently link to and activate the TRPA1 receptor, which then initiates the irritation response (Bessac and Jordt, 2010; Bautista et al., 2006, 2013). This type of irritant is exemplified by the tear gases that covalently bind to cysteine residues in the receptor (Macpherson et al., 2007; Brône et al., 2008) and can cause sustained activation of TRPA1 (Macpherson et al., 2007).

Chemicals in the non-reactive group, for example, alcohols, alkylbenzenes, non-reactive ketones, and tetrahydrocannabinol (THC) (Jordt et al., 2004), appear to physically but not covalently interact with the receptors and are not as potent as the reac- tive irritants. For example, THC activates TRPA1 presumably by binding to the active site of TRPA1 (Baraldi et al., 2010).

The epithelial cells lining the airways express the CYP450 family of enzymes that can metabolize some chemicals to reactive metabolites (Lanosa et al., 2010) that bind to TRPA1. For example, styrene and naphthalene, which are non-reactive, require metabolism to bind to the receptor and elicit the subsequent reduction in respiratory rate in mice. Differences in the CYP450 family of enzymes between humans and mice may contribute to different responses of mice and humans to some irritants (Lanosa et al., 2010).

\subsection{Key events}

\subsection{1 $\mathrm{KE}_{1}$ : Increase in intracellular $\mathrm{Ca}^{2+}$}

The binding of irritant chemicals to TRPA1 activates the ion channel, resulting in an influx of $\mathrm{Ca}^{2+}$ and an increase in intracellular $\mathrm{Ca}^{2+}$ levels as measured in cells by fluorescence dye imaging, e.g. using Fura-2 (Bessac and Jordt, 2010; Brône et al., 2008; Bessac et al., 2009). For reactive irritants, the activation depends on the covalent binding of the chemical to cysteine residues in the active site.

The increase in $\mathrm{Ca}^{2+}$ influx detected upon incubation with reactive irritants is not observed with mutant TRPA1 proteins in which the three cysteine residues in the active site have been substituted. In contrast, upon incubation of these mutant TRPA1 proteins with non-reactive irritants such as $\mathrm{THC}, \mathrm{Ca}^{2+}$ influx is

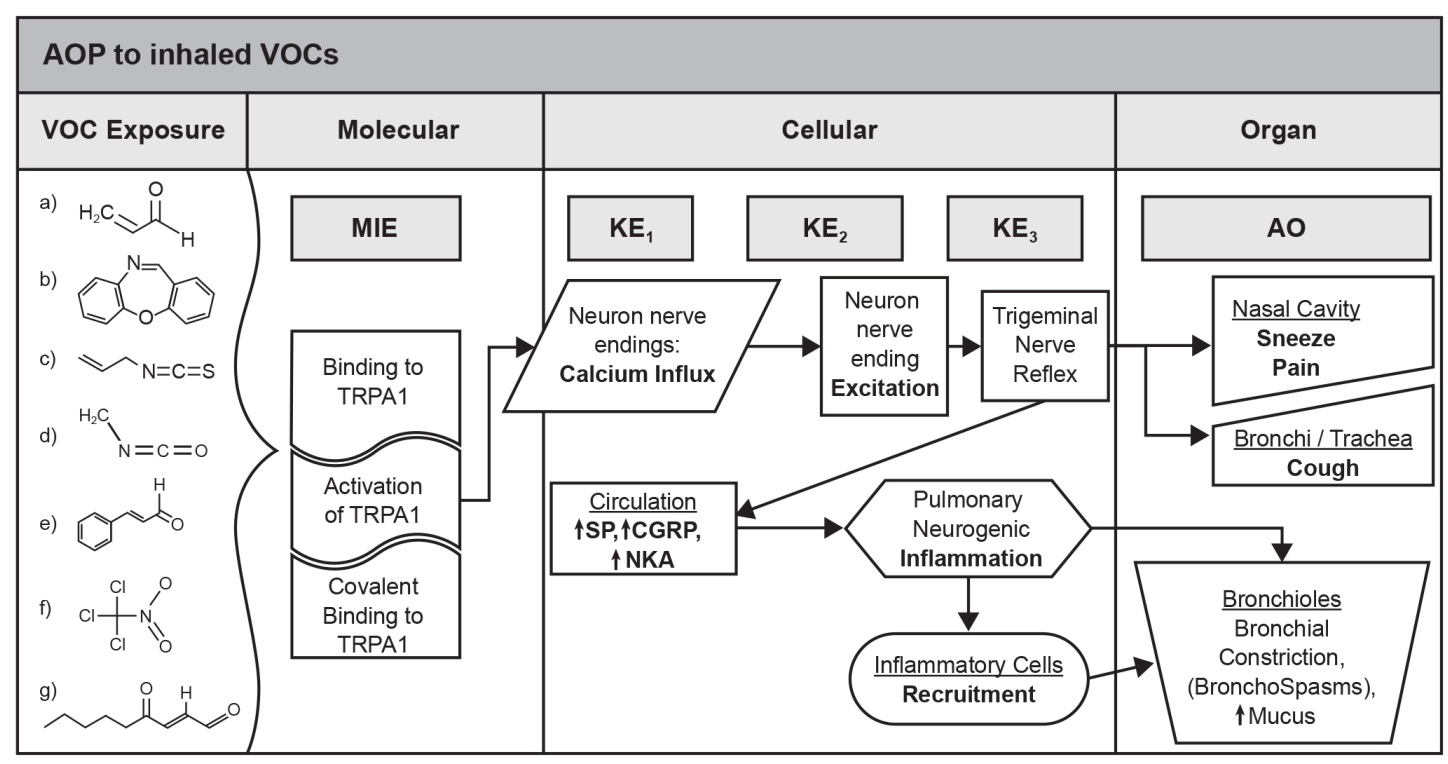

Fig. 2: Adverse outcome pathway for sensory irritation

Some examples of volatile irritant organic chemicals (VOC) are shown: a) acrolein; b) dibenz[b,f,][1,4]oxazepine(CN); c) cinnamaldehyde; d) methyl isocyanate; e) allyl-isothiocyanate; f) chloropicrin; g) 4-oxo-nonenal. 
still observed (Peterlin et al., 2007; Macpherson et al., 2007). Both covalent and non-covalent binding of chemical irritants to TRPA 1 therefore causes activation of TRPA 1 as measured by an increase in intracellular $\mathrm{Ca}^{2+}\left(\mathrm{KER}_{1}\right)$ shown in Figure 2.

In HEK-293 cells expressing TRPA1, a clear concentration dependence was found and the $\mathrm{Ca}^{2+}$ influx also increased with time of exposure to the irritant chemicals, indicating a time dependence. The $\mathrm{EC}_{50}$, i.e., the concentration causing $50 \%$ of the maximum increase in $\mathrm{Ca}^{2+}$ influx into cells, is reported for approximately 65 chemicals in the Guide to Pharmacology database with data given as $\mathrm{pEC}_{50}$, i.e., the negative $\log$ molar concentration causing 50\% of maximum response (Liu et al., 2017). Differences among rat, mouse, and human TRPA1 are also reported for some chemicals. In mice and cultured cells, the influx of $\mathrm{Ca}^{2+}$ also increased with increasing chemical concentration and time of exposure (Bessac and Jordt, 2008, 2010; Bautista et al., 2006; Brône et al., 2008).

\subsubsection{KE2: Trigeminal nerve excitation}

Electrophysiological changes occur upon an increase in intracellular $\mathrm{Ca}^{2+}$ that can lead to depolarization of sensory neurons, i.e., excitation $\left(\mathrm{KER}_{2}\right)$ (Jordt et al., 2004). Challenge of dorsal root ganglia (DRG neurons) isolated from rats with tear gases, thioisocyanate, or THC (Jordt et al., 2004; Brône et al., 2008) or challenge of isolated rat and mouse sensory neurons (DRG and nodose neurons) with reactive irritants, oxidants, or reactive lipids (Andersson et al., 2008) resulted in changes in $\mathrm{Ca}^{2+}$ influx and changes in membrane potential that were both concentration- and time-dependent.

Furthermore, mouse DRG neurons isolated from TRPA1-/mice have greatly reduced increases in $\mathrm{Ca}^{2+}$ influx and electrophysiological changes after challenge with several irritants as compared to DRG isolated from wild-type mice. In contrast, DRG neurons isolated from TRPV1-/- have responses similar to DRG neurons isolated from wild-type mice after stimulation with irritants. After exposure to inhaled irritants, TRPA1-/- mice but not TRPV1-/- mice displayed significantly lesser trigeminal/ vagal responses than wild-type mice as measured by respiration rate and symptoms of irritation, i.e., pain, coughing, sneezing. Studies with TRPA1-/- mice strongly support the critical importance of trigeminal excitation after activation of TRPA1 receptor in response to irritants (Bessac and Jordt, 2008, 2010; Bautista et al., 2006; Brône et al., 2008).

The reduction in respiratory rate after exposure to chemicals at different concentrations measured as reported by Alarie et al. (1981, 1995) can be considered an estimate of trigeminal nerve excitation in mice after exposure. Studies with TRPA1-/- mice support the hypothesis that trigeminal excitation is dependent on TRPA1. The decrease in respiration rate occurs with increasing chemical concentration, supporting the sensory assay $\left(\mathrm{RD}_{50}\right)$ correlation of trigeminal nerve excitation in vivo.

\subsection{3 $\mathrm{KE}_{3}$ : Neurogenic inflammation}

Trigeminal excitation of chemosensory nerve endings in the nasal mucosa and airways after activation of TRPA 1 stimulates the release of the neurogenic inflammatory neuropeptides SP and
CGRP $\left(\mathrm{KER}_{3}\right)$, which promote neurogenic inflammation, vasodilation and fluid leakage. SP stimulates goblet cells and submucosal glands to increase mucus secretion and contractility of airway smooth muscles, which results in bronchial constriction and increased airway resistance (O'Connor et al., 2004). CGRP stimulates the pulmonary vasculature, causing plasma extravasation, edema, and neutrophil infiltration (Andrè et al., 2008; Trevisani et al., 2007). Clear time- and concentration-dependent relationships between activation of the receptor, $\mathrm{Ca}^{2+}$ ion flux and the release of neuropeptides are reported (Bessac and Jordt, 2010, 2008).

TRPA1-/- mice, but not TRPV1-/- mice, show greatly reduced leukocyte infiltration, reduced cytokine and mucus production, and abolished hyperactivity and hence impaired inflammation and hyper-reactivity in comparison to wildtype mice. The lack of an irritant response and reduced neurogenic inflammation after exposure to known irritants in TRPA1-/- mice provides compelling evidence supporting the linkage of TRPA1 activation and neuropeptide release (Bautista et al., 2006; Caceres et al., 2009).

\subsection{The adverse outcome}

Excitation of the trigeminal nerves causes the initiation of airway reflex responses, coughing, sneezing, and pain. The release of inflammatory neuropeptides induces bronchoconstriction, vasodilation, recruitment of immune cells, and an inflammatory response (Bautista et al., 2006, 2013). As the irritant reaches the lower airways, sensory nerve activation causes the following organ or pulmonary responses: bronchial constriction spasms, increased mucus production, and further neurogenic inflammation. Increased eosinophils, $\mathrm{T}$ helper cells and release of the associated inflammatory cytokines (IL-2, IL-4, IL-10, IL-13) are observed (Caceres et al., 2009; Belvisi et al., 2011).

Trigeminal activation also results in a vagal response that slows the respiration rate (Alarie, 1981; Alarie et al., 1995). Exposure to irritants and the activation of TRPA1 coupled with its interaction with TRPV1 can eventually lead to the following organism responses or clinical manifestations: chronic cough, pain, airway inflammation, COPD, and asthmatic-like conditions (Bessac and Jordt, 2008; Chen and Hackos, 2015; Baraldi et al., 2010). The activation of these receptors causes airway hyperresponsiveness and induction of neurogenic inflammation (Bessac and Jordt, 2010; Belvisi et al., 2011).

The activation of TRPA1 and TRPV1 induces the release of pro-inflammatory peptides such as NG, SP, and CGRP that mediate neurogenic inflammation observed as bronchoconstriction, vasodilation, and the recruitment of immune cells (Bautista et al., 2006, 2013).

\subsection{Weight of evidence assessment for the AOP}

Evaluation of the AOP was based on the Bradford Hill criteria as described in the OECD AOP Handbook (OECD, 2012a,b, 2018) with the additional guidance provided in Becker et al. (2015) and Vinken et al. (2013). A box and linear flow diagram (Fig. 1) was constructed to allow easy determination of the sequence of biological events at the different levels of biological organization. 
The AOP was evaluated based on the weight of evidence (OECD, 2012a, 2018) for the following criteria: 1) dose-response relationships, 2) temporal relationship between the key events and adverse effect, 3) strength, consistency, and specificity between adverse effect and initiating event, 4) biological plausibility, coherence, and consistency of the experimental evidence, 5) alternative mechanisms that logically present themselves and the extent to which they may distract from the postulated AOP, and 6) uncertainties, inconsistencies, and missing data.

\subsubsection{Molecular initiating event (MIE)}

The MIE is the binding of the chemical to the TRPA1 receptor, which results in an increase in intracellular $\mathrm{Ca}^{2+}$. The importance of the activation of the TRPA1 receptor in the pulmonary response to irritants is strongly supported by the results from studies with knockout mice, i.e., TRPA1-/- mice and TRPV-/- mice (Taylor-Clark et al., 2009; Bessac et al., 2008, 2009; Caceres et al., 2009). For TRPA1-/- mice, a diminished response to irritant chemicals is reported, confirming the association of the initiating event, i.e., the activation of the TRPA1 receptor, with the adverse effect. In contrast, after exposure to irritant chemicals, the irritation response was the same in TRPV1-/- mice compared to wild-type mice, consistent with the high specificity between TRPA1 activation and the adverse effect. Actual binding of the reactive chemicals to TRPA1 was confirmed by mass spectrum analysis. However, no dose-response was measured for the binding (Macpherson et al., 2007; Brône et al., 2008). The studies in knockout mice provide a high strength, consistency, and specificity for the association of the initiating event with the adverse outcome.

$\mathrm{Ca}^{2+}$ influx determination by Fura-2 fluorescence measurement in fibroblasts expressing TRPA1 is a well-established in vitro assay to measure the activation of TRPA1 by irritants. Many investigators have used this assay to measure the potency of selected irritant chemicals from with the $\mathrm{pEC}_{50}$ values reported in the Guide to Pharmacology are derived (Liu et al., 2017). A commonly used fibroblast cell line is HEK-293, which can be transfected readily with TRPA1 and is conducive to $\mathrm{Ca}^{2+}$ measurement. As a human transformed lung epithelial cell line, A549 offers the advantage of already expressing functional TRPA1 (Buch et al., 2013).

In vitro data on the activation of TRPA1 by known irritants as measured directly in cells expressing TRPA1 causes some uncertainty in the MIE since only a few irritants have been tested both in vitro and in vivo. In general, the potency of a few irritants obtained from in vitro assays for the activation of TRPA1 is in agreement with the concentrations required to produce adverse effects in mice (Bessac et al., 2008, 2009; Brône et al., 2008; McNamara et al., 2007; Schaper, 1993). The correlation between the potency of most irritants as measured by in vitro methods and the concentrations required for the irritation or the adverse effects in mice has not been fully investigated.

While the weight of evidence for activation of TRPA1 by chemicals in the mouse is high, with a high confidence level, the exact mechanism and interactions with other receptors present in the trigeminal ganglia neurons are less clear. In general, howev- er, there is a high strength, consistency, and specificity for the association of adverse effects and the initiating event.

\subsection{2 $\mathrm{KE}_{1}$ and $K E R_{1}$}

TRPA1 is a member of the TRP family of $\mathrm{Ca}^{2+}$ ion channels and a strong coherence and consistency exists with the experimental evidence that the binding of the chemicals to TRPA1 results in an increase in intracellular $\mathrm{Ca}^{2+}$ (Bessac and Jordt, 2008, 2010; Bautista et al., 2006; Brône et al., 2008). Studies with TRPA1-/cells and mice confirm that TRPA1 is essential for the increase in intracellular $\mathrm{Ca}^{2+}$ observed after challenge with irritant chemicals. Concordances between dose responses and temporal relationships were observed in vitro for increased intracellular $\mathrm{Ca}^{2+}$ and the trigeminal nerve excitation as measured by changes in electric potential (Bessac and Jordt, 2008; Bessac et al., 2008). The weight of evidence for both $\mathrm{KE}_{1}$ and $\mathrm{KER}_{1}$ is high, and the confidence level is high.

\subsection{3 $\mathrm{KE}_{2}$ and $\mathrm{KER}_{2}$}

The temporal sequence of events and the concentration dependency for intracellular $\mathrm{Ca}^{2+}$ agree with the electro-physiological changes observed in cells and isolated trigeminal neurons after irritant chemical challenge (Bessac and Jordt, 2008, 2010; Bautista et al., 2006; Brône et al., 2008). The results from experiments with site-directed mutagenesis confirm the dependence of the electric potential differences, and hence the nerve excitation, by TRPA 1 on $\mathrm{Ca}^{2+}$ ion influx (Doerner et al., 2007). Other studies with TRPA1-/- mice confirm that the presence of TRPA1 is essential for the trigeminal nerve excitation and irritation in mice (Bessac and Jordt, 2008, 2010; Bautista et al., 2006; Brône et al., 2008; Achanta and Jordt, 2017), providing biological plausibility, coherence, and consistency with a high confidence level in $\mathrm{KE}_{2}$ and $\mathrm{KER}_{2}$.

\subsection{4 $\mathrm{KE}_{\mathbf{3}}$ and $\mathrm{KER}_{\mathbf{3}}$}

Trigeminal nerve excitation stimulates the release of the pro-inflammatory neuropeptides SP and CGRP, which cause neurogenic inflammation (Trevisanti et al., 2007; Andrè et al., 2008; Caceres et al., 2009). Trigeminal nerve-mediated neurogenic inflammation causes excitation of the sneezing, pain, coughing, and vagal stimulation, resulting in a decreased rate of respiration. These biological responses to irritant exposure are diminished in TRPA1-/- mice, confirming the essential role of TRPA1 in the adverse outcome, i.e., sensory pulmonary irritation (Bessac et al., 2008; Bautista et al., 2006; Taylor-Clark et al., 2009; Liu et al., 2013; McNamara et al., 2007). Other studies with TRPA1-/- mice confirm the essential role of TRPA1 in airway inflammation, hyperreactivity, and mast cell induction (Hox et al., 2013; Caceres et al., 2009).

The temporal sequence of biochemical and physiological events agrees with the adverse outcome of sensory pulmonary irritation as observed at different times after exposure (Bessac and Jordt, 2008, 2010; Bautista et al., 2006; Brône et al., 2008). The dose-response relationship between the activation of TRPA1 and neurogenic inflammation based on in vitro and in vivo experimental evidence is consistent with the hypothesis that the ac- 
tivation of the TRPA1 receptor is a key initiation event in sensory pulmonary irritation (Bautista et al., 2006, 2013; Bessac and Jordt, 2008; Belvisi et al., 2011). Biological plausibility is high and consistent with other studies on neurogenic inflammation (Meseguer et al., 2014; Xanthos and Sandkuhler, 2014), with strong coherence and consistency of the experimental evidence to support the essential role, and empirical results indicating that the weight of evidence and confidence in $\mathrm{KE}_{3}$ and $\mathrm{KER}_{3}$ is high.

\subsubsection{Weight of evidence for AOP}

The analysis of the overall weight of evidence for this AOP is strong and there is a high level of confidence based on Bradford-Hill criteria for the analysis of the key events and the KERs connecting the key events to the adverse outcome sensory pulmonary irritation. The biological plausibility based on the experimental evidence is strong for the KEs and KERs based on the knowledge obtained from numerous investigations. The concordance of dose responses and temporal relationships also strongly supports the high confidence level. Evidence of essentiality for the AOP is based on diminished response to irritant chemicals observed with TRPA1-/- mice. All the key events and the adverse outcome were dependent on the MIE, i.e., the activation of TRPA1.

\section{Discussion}

There is compelling evidence as reported in the literature by several investigators (Bautista et al., 2013; Bessac and Jordt, 2010; Baraldi et al., 2010) to support this sequence of key biological events in the AOP. Activation of the TRPA1 receptor is the critical event; it is the MIE that initiates this series of biological events causing respiratory irritation. The concentration dependence for the activation of TRPA1 by irritant chemicals determines, in part, its potency to cause biological effects in vitro or in animal models.

More than 350 chemicals characterized as pulmonary irritants have been investigated with a mouse assay for sensory pulmonary irritation based on measurement of changes in respiration (Alarie, 1981; Alarie et al., 1995) with the data reported as $\mathrm{RD}_{50}$ values (Schaper, 1993). Excitation of the trigeminal nerves after activation of TRPA1 by an inhaled irritant causes a vagal-mediated reduction in respiration rate. The reduction in respiration rate is attenuated in TRPA1-/- mice in response to exposure to an inhalable sensory pulmonary irritant (Bessac and Jordt, 2010; Achanta and Jordt, 2017), providing evidence that reduction in respiration rate is a measure of TRPA 1 activation. The $\mathrm{RD}_{50}$ values may be considered an indirect measure of potency of an irritant to stimulate trigeminal nerve activation in mice, but this in vivo assay is compromised by various issues associated with animal experiments, i.e., pharmacokinetic issues, volatility of the chemicals, etc.

An apparent correlation is reported between $\mathrm{RD}_{50}$ values and threshold limit values (TLVs) for some of these chemicals. $\mathrm{Ku}-$ wabara et al. (2007) found relationships between $\mathrm{RD}_{50}$ values and the lowest observed adverse effect levels (LOAELs) and acute exposure reference levels for 16-25 irritant chemicals. They concluded that $\mathrm{RD}_{50}$ data are useful for setting protective exposure levels for both workers and the general population. Sensory irritation has been proposed as a basis for setting occupational limits (Brüning et al., 2014; Nielsen and Wolkoff, 2017). Recently, Nielsen and Wolkoff et al. (2017) evaluated the mouse bioassay for setting exposure limits or guidelines for exposure to airborne irritants. They concluded this assay was the "only validated animal bioassay for prediction of sensory irritation in humans." Exposure levels and $\mathrm{RD}_{50}$ data appear to be linked to the critical biochemical event (MIE) determined by the AOP.

For some irritant chemicals, including a few environmental chemicals, the activation of TRPA1 has been determined in HEK-293T fibroblasts and other cells that express TRPA1. Known irritant chemicals (65 are reported) bind to and activate TRPA1 (Liu et al., 2017). Although many VOCs are characterized as pulmonary irritants, only a few of these environmental irritants have been tested using in vitro assays to directly measure the activation of TRPA1 and only a limited number of $\mathrm{EC}_{50}$ are reported (Bessac and Jordt, 2010; Bos et al., 1991, 2002; Lehmann et al., 2016).

Based on the understanding of respiratory irritation from the AOP and the data showing that the mouse sensory assay indirectly measures TRPA1 activation, we propose to use the in vitro assay based on fibroblasts or other cells expressing TRPA1 to measure the direct activation of TRPA1 by suspected irritants. The assay could be used to screen chemicals for the activation of TRPA1 and to determine the concentration dependence or $\mathrm{EC}_{50}$. The in vitro assay could be used with other assays to help set exposure limits. A comparison of the $\mathrm{RD}_{50}$ values with the $\mathrm{EC}_{50}$ values is a suitable approach to test the hypothesis that the $\mathrm{EC}_{50}$ values and $\mathrm{RD}_{50}$ values correlate.

To identify existing $\mathrm{EC}_{50}$ values for sensory irritants, the primary references identified in our extensive literature search and data reported in the Guide to Pharmacology database were examined (Liu et al., 2017). The $\mathrm{pEC}_{50}$ values were cross-referenced with the chemicals reported with the Schaper database (1993). Only 7 chemicals with both $\mathrm{RD}_{50}$ and $\mathrm{pEC}_{50}$ values were identified that had 2015 TLVs (threshold limit value) developed based solely on respiratory irritation by the American Conference of Governmental Industrial Hygienists (ACGIH). A preliminary analysis of this published data was done to determine the feasibility of this approach and is reported in Figure 3 and Table 1 . The result shows a linear relationship between the in vivo $\mathrm{RD}_{50}$ and in vitro $\mathrm{pEC}_{50}$ values and is support for this proposal. The results suggest that $\mathrm{pEC}_{50}$ values for the activation of TRPA1 obtained from the in vitro assay may be used to estimate in vivo $\mathrm{RD}_{50}$ or potentially replace the mouse sensory assay and hence be used in setting exposure levels of irritant chemicals. Additional support for this proposal is the good correlation that exists between the potency of tear gases determined by an in vitro assay using human TRPA1 expressed in cells and the concentrations causing $50 \%$ of exposed human subjects to detect the tear gas or to become incapacitated by the exposure (Brône et al., 2008). The development of a validated in vitro assay for TRPA1 activation can be used to determine if an uncharacterized chem- 


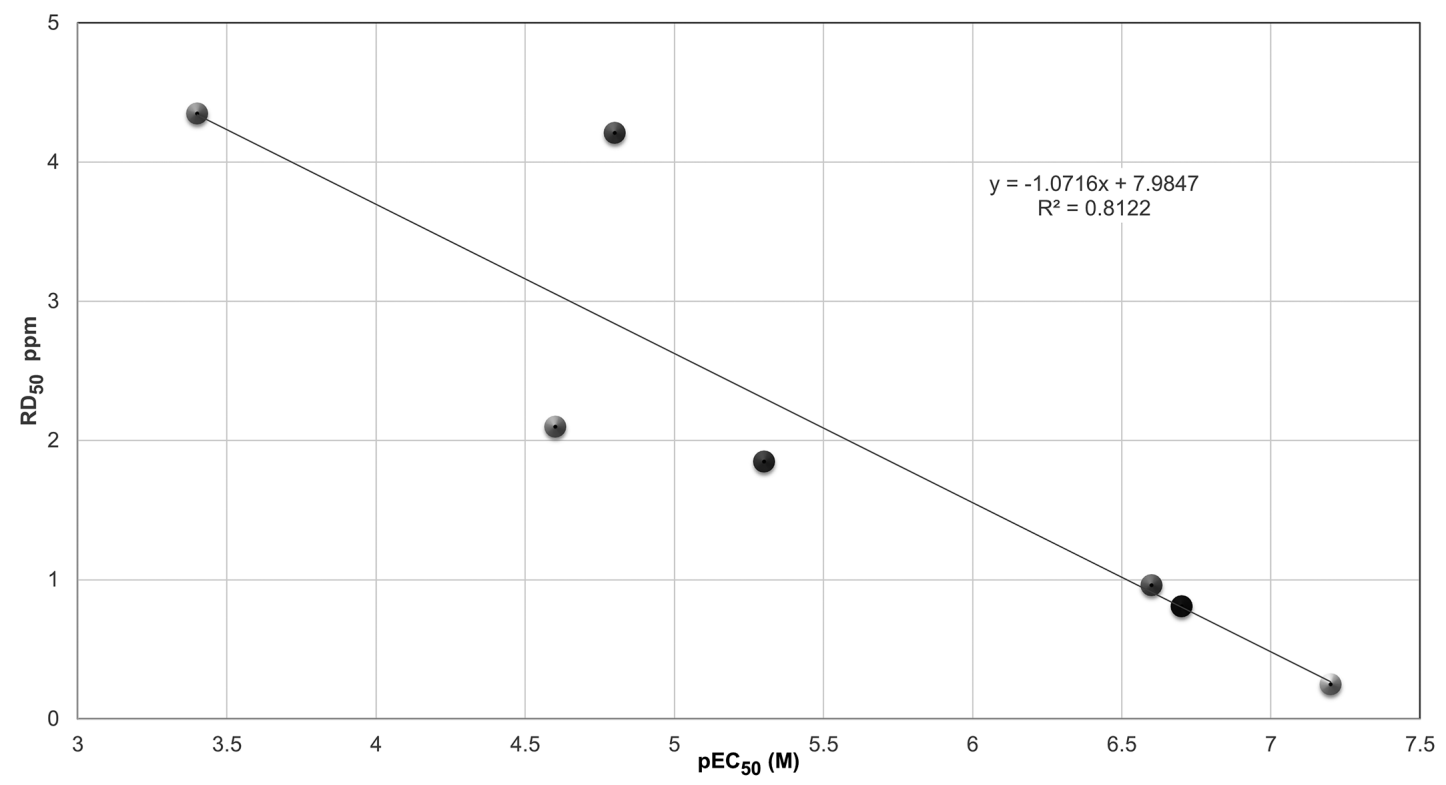

Fig. 3: The relationship between the $\mathrm{RD}_{50}$ values reported for exposure of mice to irritants and the reported $\mathrm{pEC} \mathrm{C}_{50}$ for the in vitro activation of TRPA1

The vertical linear axis shows the $\mathrm{RD}_{50}(\mathrm{ppm})$ values as reported for mice. The horizontal log axis shows $\mathrm{pEC} \mathrm{C}_{50}(\mathrm{M})$ values for the activation of TRPA1 obtained by measuring the changes in $\mathrm{Ca}^{2+}$ flux in fibroblasts expressing human TRPA1 as reported in Liu et al. (2017). All $\mathrm{pEC}_{50}$ are from cells expressing human TRPA1 except for formaldehyde obtained with mouse TRPA1 and crotonaldehyde obtained with rat TRPA1. The chemicals and data are listed in Table 1.

Tab. 1: Volatile organic chemicals (VOCs) used to compare in vivo $\mathrm{RD}_{50}$ values (mean $\pm \mathrm{SD}$ ) with in vitro $\mathrm{pEC}$ (50 values For the $\mathrm{RD}_{50}$ values, mice were exposed for 3-10 minutes. The $\mathrm{pEC}_{50}$ (negative logarithm to base 10 of the concentration) exposure time to produce the maximal response is typically seconds to minutes.

\begin{tabular}{|l|l|l|l|}
\hline Chemical $^{\mathrm{a}}$ & CAS RN & RD $_{50}{ }^{\mathrm{b}, \mathrm{c}}(\mathbf{p p m})$ & $\mathbf{p E C}_{50}{ }^{\mathrm{d}}(\mathbf{M})$ \\
\hline Formaldehyde & $50-00-0$ & $4.35 \pm 0.94(\mathrm{n}=4)$ & 3.4 \\
\hline Methyl isocyanate (MIC) & $624-83-9$ & $2.1 \pm 1.13(\mathrm{n}=2)$ & 4.6 \\
\hline Crotonaldehyde & $123-73-9$ & $4.21 \pm 0.95(\mathrm{n}=2)$ & 4.8 \\
\hline Acrolein & $107-02-8$ & $1.85 \pm 0.69(\mathrm{n}=7)$ & 5.3 \\
\hline 1-Chloroacetophenone (CN) & $532-27-4$ & 0.96 & 6.6 \\
\hline 2-Chlorobenzyl-malononitrile (CS) & $2698-41-1$ & $0.81 \pm 0.54(\mathrm{n}=2)$ & 6.7 \\
\hline Dibenz[b,f,][1,4]-oxazepine (CR) & $257-07-8$ & 0.25 & 7.2 \\
\hline
\end{tabular}

${ }^{a}$ Chemicals with both $\mathrm{RD}_{50}$ and $\mathrm{pEC}_{50}$ values were identified that had 2015 TLVs (threshold limit value) developed by the American Conference of Governmental Industrial Hygienists (ACGIH) based solely on respiratory irritation. The TLV is a level to which a worker can be exposed day after day for a working lifetime without adverse effects. Chemicals with TLVs based upon pulmonary sensitization or edema were excluded due to the complexity of multiple mechanisms. ${ }^{\text {b}}$ Schaper, 1993; ${ }^{\mathrm{C}}$ Alarie, 2015; ${ }^{\mathrm{d}}$ Liu et al., 2017 
ical is a potential chemical irritant. The results obtained may be useful as predictive tests and ultimately for setting human inhalation exposure limits.

Confirming the in vitro activation of TRPA1 by other established irritants would enhance the scope of this AOP. If some of these established irritants were found not to be activators of TRPA1 receptors, then fibroblasts expressing TRPV1 or ASIC may be appropriately used. TRPA1 and TRPV1 co-localize in the nerve ending (Bessac and Jordt, 2008) and some data suggest possible interactions between these two receptors. For example, the diallyl sulfides present in garlic activate both the TRPV1 and TRPA1, but activation of TRPA1 was observed at lower concentrations (Koizumi et al., 2009). Lehmann et al. (2016, 2017) have also provided evidence of activation of both TRPA1 and TRPV1 in response to 2-ethyl hexanol in vitro and suggested that the use of several in vitro assays is necessary to investigate and fully characterize irritants. Fibroblasts or oocytes constructed to co-express both TRPA1 and TRPV1 can be used to determine whether their interaction alters the $\mathrm{EC}_{50}$.

Primary cultures of trigeminal neurons and calcium imaging have also been used to investigate activation by a series of VOC irritants (Inoune and Bryant, 2005; Lehmann et al., 2016, 2017). The activation of the neurons did not always agree with the respective $\mathrm{RD}_{50}$ value. This suggests that additional mechanisms may also be responsible for the irritation observed in the mouse assay.

In addition, using the in vitro assay combined with other assays to measure the biochemical and cellular processes described as the key events of this AOP (the increase in intracellular $\mathrm{Ca}^{2+}$, trigeminal nerve excitation measured by changes in cellular membrane potential or calcium imaging, and measurements of the release of neurogenic pro-inflammatory neuropeptides or cytokines) as a multitiered approach could increase the predictive accuracy and requires further research.

In summary, the development of an AOP for sensory pulmonary irritation provides evidence that the activation of TRPA1 is the MIE that results in an increase in intracellular $\mathrm{Ca}^{2+}$ leading to trigeminal nerve excitation and neurogenic inflammation to cause the adverse outcome of sensory pulmonary irritation. This understanding leads to a proposed approach to use a cellular-based in vitro assay to directly measure the activation of TRPA1 to determine if a suspected chemical is an irritant and then to determine its potency. With further development and validation this in vitro assay to measure the activation of TRPA1 may be a suitable replacement for the mouse sensory $\mathrm{RD}_{50}$ assay that can be used to set inhalation exposure limits.

\section{References}

Achanta, S. and Jordt, S. E. (2017). TRPA1: Acrolein meets its target. Toxicol Appl Pharmacol 324, 45-50. doi:10.1016/j.taap. 2017.03.007

Alarie, Y. (1966). Irritating properties of airborne materials to the upper respiratory tract. Arch Environ Health 13, 433-449.

Alarie, Y. (1973). Sensory irritation by airborne chemicals. CRC Crit Rev Toxicol 2, 299-363. doi:10.3109/10408447309082020
Alarie, Y. (1981). Bioassay for evaluating the potency of airborne sensory irritants and predicting acceptable levels of exposure in man. Food Cosmet Toxicol 19, 623-626. doi:10.1016/00156264(81)90513-7

Alarie, Y., Nielsen, G. D., Andonian-Haftvan, J. and Abraham, M. H. (1995). Physicochemical properties of nonreactive volatile organic chemicals to estimate $\mathrm{RD}_{50}$ : Alternatives to animal studies. Toxicol Appl Pharmacol 134, 92-99. doi:10.1006/ taap.1995.1172

Alarie, Y. E. (2015). Table 1. 2015 Update of the 1993 Schaper database of $\mathrm{RD}_{50}$ and their TLV values. http://www.pitt. edu/ rd50/ (accessed November 2018).

Andersson, D. A., Gentry, C., Moss, S. and Bevan, S. (2008). Transient receptor potential A1 is a sensory receptor for multiple products of oxidative stress. $J$ Neurosci 28, 2485-2494. doi:10.1523/JNEUROSCI.5369-07.2008

Andrè, E., Campi, B., Materazzi, S. et al. (2008). Cigarette smoke-induced neurogenic inflammation is mediated by alpha,beta-unsaturated aldehydes and the TRPA1 receptor in rodents. J Clin Invest 118, 2574-2582. doi:10.1172/JCI34886

Baraldi, P. G., Preti, D., Materazzi, S. and Geppetti, P. (2010). Transient receptor potential ankyrin 1 (TRPA1) channel as emerging target for novel analgesics and anti-inflammatory agents. J Med Chem 53, 5085-5107. doi:10.1021/jm100062h

Bautista, D. M., Jordt, S.-E., Nikai, T. et al. (2006). TRPA1 mediates the inflammatory actions of environmental irritants and proalgesic agent. Cell 124, 1269-1282. doi:10.1016/j.cell. 2006.02.023

Bautista, D. M., Pellegrino, M. and Tsunozaki, M. (2013). TRPA1: A gatekeeper for inflammation. Annu Rev Physiol 75, 181-200. doi:10.1146/annurev-physiol-030212-183811

Becker, R. A., Ankley, G. T., Edwards, S. W. et al. (2015). Increasing scientific confidence in adverse outcome pathways: Application of tailored bradford-hill considerations for evaluating weight of evidence. Regul Toxicol Pharmacol 72, 514537. doi:10.1016/j.yrtph.2015.04.004

Belvisi, M. G., Dubuis, E. and Birrell, M. A. (2011). Transient receptor potential A1 channels: Insights into cough and airway inflammatory disease. Chest 140, 1040-1047. doi:10.1378/ chest.10-3327

Bessac, B. F. and Jordt, S. E. (2008). Breathtaking TRP channels: TRPA1 and TRPV1 in airway chemosensation and reflex control. Physiology (Bethesda) 23, 360-370. doi:10.1152/ physiol.00026.2008

Bessac, B. F., Sivula, M., von Hehn, C. A. et al. (2008). TRPA1 is a major oxidant sensor in murine airway sensory neurons. $J$ Clin Invest 118, 1899-1910. doi:10.1172/JCI34192

Bessac, B. F., Sivula, M., von Hehn, C. A. et al. (2009). Transient receptor potential ankyrin 1 antagonists block the noxious effects of toxic industrial isocyanates and tear gases. FASEB J 23, 1102-1114. doi:10.1096/fj.08-117812

Bessac, B. F. and Jordt, S. E. (2010). Sensory detection and responses to toxic gases: Mechanisms, health effects, and countermeasures. Proc Am Thorac Soc 7, 269-277. doi:10.1513/pats.201001-004SM

Bos, P. M., Zwart, A., Reuzel, P. G. and Bragt, P. C. (1991). 
Evaluation of the sensory irritation test for the assessment of occupational health risk. Crit Rev Toxicol 21, 423-450. doi:10.3109/10408449209089882

Bos, P. M., Busschers, M. and Arts, J. H. (2002). Evaluation of the sensory irritation test (Alarie test) for the assessment of respiratory tract irritation. J Occup Environ Med 44, 968-976. doi:10.1097/00043764-200210000-00017

Brône, B., Peeters, P. J., Marrannes, R. et al. (2008). Tear gasses $\mathrm{CN}, \mathrm{CR}$, and $\mathrm{CS}$ are potent activators of the human TRPA1 receptor. Toxicol Appl Pharmacol 231, 150-156. doi:10.1016/j. taap.2008.04.005

Brooks, S. M., Weiss, M. A. and Bernstein, I. L. (1985). Reactive airways dysfunction syndrome (RADS). Persistent asthma syndrome after high level irritant exposures. Chest 88, 376384. doi:10.1378/chest.88.3.376

Brüning, T., Bartsch, R., Bolt, H. et al. (2014). Sensory irritation as a basis for setting occupational exposure limits. Arch Toxicol 88, 1855-1879. doi:10.1007/s00204-014-1346-Z

Buch, T. R., Schafer, E. A., Demmel, M. T. et al. (2013). Functional expression of the transient receptor potential channel TRPA1, a sensor for toxic lung inhalants, in pulmonary epithelial cells. Chem Biol Interact 206, 462-471. doi:10.1016/j. cbi.2013.08.012

Caceres, A. I., Brackmann, M., Elia, M. D. et al. (2009). A sensory neuronal ion channel essential for airway inflammation and hyperreactivity in asthma. Proc Natl Acad Sci U S A 106, 9099-9104. doi:10.1073/pnas.0900591106

Chen, J. and Hackos, D. H. (2015). TRPA1 as a drug target Promise and challenges. Naunyn Schmiedebergs Arch Pharmacol 388, 451-463. doi:10.1007/s00210-015-1088-3

Doerner, J. F., Gisselmann, G., Hatt, H. and Wetzel, C. H. (2007). Transient receptor potential channel A1 is directly gated by calcium ions. J Biol Chem 282, 13180-13189. doi:10.1074/ jbc.M607849200

Fujita, F., Uchida, K., Moriyama, T. et al. (2008). Intracellular alkalization causes pain sensation through activation of TRPA1 in mice. J Clin Invest 118, 4049-4057. doi:10.1172/JCI35957

Hox, V., Vanoirbeek, J. A., Alpizar, Y. A. et al. (2013). Crucial role of transient receptor potential ankyrin 1 and mast cells in induction of nonallergic airway hyperreactivity in mice. Am J Respir Crit Care Med 187, 486-493. doi:10.1164/rccm. 201208-13580C

Inoue, T. and Bryant, B. P. (2005). Multiple types of sensory neurons respond to irritating volatile organic compounds (VOCs): Calcium fluorimetry of trigeminal ganglion neurons. Pain 117, 193-203. doi:10.1016/j.pain.2005.06.012

Jordt, S. E., Bautista, D. M., Chuang, H. H. et al. (2004). Mustard oils and cannabinoids excite sensory nerve fibres through the TRP channel ANKTM1. Nature 427, 260-265. doi:10.1038/ nature 02282

Kichko, T. I., Kobal, G. and Reeh, P. W. (2015). Cigarette smoke has sensory effects through nicotinic and TRPA1 but not TRPV1 receptors on the isolated mouse trachea and larynx. Am J Physiol Lung Cell Mol Physiol 309, L812-820. doi:10.1152/ajplung.00164.2015

Koizumi, K., Iwasaki, Y., Narukawa, M. et al. (2009). Dial- lyl sulfides in garlic activate both TRPA1 and TRPV1. Biochem Biophys Res Commun 382, 545-548. doi:10.1016/j. bbrc.2009.03.066

Kuwabara, Y., Alexeeff, G. V., Broadwin, R. and Salmon, A. G. (2007). Evaluation and application of the $\mathrm{RD}_{50}$ for determining acceptable exposure levels of airborne sensory irritants for the public. Environ Health Perspect 115, 1609-1616. doi:10.1289/ehp.9848

Lanosa, M. J., Willis, D. N., Jordt, S. and Morris, J. B. (2010). Role of metabolic activation and the TRPA1 receptor in the sensory irritation response to styrene and naphthalene. Toxicol Sci 115, 589-595. doi:10.1093/toxsci/kfq057

Lehmann, R., Schobel, N., Hatt, H. and van Thriel, C. (2016). The involvement of TRP channels in sensory irritation: A mechanistic approach toward a better understanding of the biological effects of local irritants. Arch Toxicol 90, 1399-1413. doi:10.1007/s00204-016-1703-1

Lehmann, R., Hatt, H. and van Thriel, C. (2017). Alternative in vitro assays to assess the potency of sensory irritants - Is one TRP channel enough? Neurotoxicology 60, 178-186. doi:10.1016/j.neuro.2016.08.010

Liu, B., Escalera, J., Balakrishna, S. et al. (2013). TRPA1 controls inflammation and pruritogen responses in allergic contact dermatitis. FASEB J 27, 3549-3563. doi:10.1096/fj.13-229948

Liu, B., Fan L., Nilius B. et al. (2017). Transient receptor potential channels: TRPA1. Last modified on 15.03.2017. IUPHAR/ BPS Guide to Pharmacology, database. http://www.guide topharmacology.org/GRAC/ObjectDisplayForward?objectId $=485$ (accessed 04.03.2019).

Macpherson, L. J., Dubin, A. E., Evans, M. J. et al. (2007). Noxious compounds activate TRPA1 ion channels through covalent modification of cysteines. Nature 445, 541-545. doi:10.1038/nature 05544

McNamara, C. R., Mandel-Brehm, J., Bautista, D. M. et al. (2007). TRPA1 mediates formalin-induced pain. Proc Natl Acad Sci U S A 104, 13525-13530. doi:10.1073/pnas. 0705924104

Meseguer, V., Alpizar, Y. A., Luis, E. et al. (2014). TRPA1 channels mediate acute neurogenic inflammation and pain produced by bacterial endotoxins. Nat Commun 5, 3125. doi:10. 1038/ncomms4125

Naono-Nakayama, R., Sunakawa, N., Ikeda, T. and Nishimori, T. (2010). Differential effects of substance P or hemokinin-1 on transient receptor potential channels, TRPV1, TRPA1 and TRPM8, in the rat. Neuropeptides 44, 57-61. doi:10.1016/j. npep.2009.10.002

Nielsen, G. D. and Wolkoff, P. (2017). Evaluation of airborne sensory irritants for setting exposure limits or guidelines: A systematic approach. Regul Toxicol Pharmacol 90, 308-317. doi:10.1016/j.yrtph.2017.09.015

O’Connor, T. M., O’Connell, J., O’Brien, D. I. et al. (2004). The role of substance $\mathrm{P}$ in inflammatory disease. $J$ Cell Physiol 201, 167-80. doi:10.1002/jcp.20061

OECD (2012a). The Adverse Outcome Pathway for Skin Sensitization initiated by covalent binding to proteins Part 1: Scientific Evidence. https://bit.ly/1EEvQoq 
OECD (2012b). Proposal for a Template and Guidance on Developing and Assessing the Completeness of Adverse Outcome Pathways. http://www.oecd.org/chemicalsafety/testing/ 49963554.pdf

OECD (2018). Users' Handbook supplement to the Guidance Document for developing and assessing Adverse Outcome Pathways. OECD Series on Adverse Outcome Pathways No. 1. OECD Publishing, Paris. doi:10.1787/5jlv1m9d1g32-en

Peterlin, Z., Chesler, A. and Firestein, S. (2007). A painful trp can be a bonding experience. Neuron 53, 635-638. doi:10.1016/j. neuron.2007.02.011

Rowell, T. R. and Tarran, R. (2015). Will chronic e-cigarette use cause lung disease? Am J Physiol Lung Cell Mol Physiol 309, L1398-1409. doi:10.1152/ajplung.00272.2015

Russell, F. A., King, R., Smillie, S. J. et al. (2014). Calcitonin gene-related peptide: Physiology and pathophysiology. Physiol Rev 94, 1099-1142. doi:10.1152/physrev.00034.2013

Schaper, M. (1993). Development of a database for sensory irritants and its use in establishing occupational exposure limits. Am Ind Hyg Assoc J 54, 488-544. doi:10.1080/ 15298669391355017

Symanowicz, P. T., Gianutsos, G. and Morris, J. B. (2004). Lack of role for the vanilloid receptor in response to several inspired irritant air pollutants in the $\mathrm{C} 57 \mathrm{Bl} / 6 \mathrm{~J}$ mouse. Neurosci Lett 362, 150-153. doi:10.1016/j.neulet.2004.03.016

Taylor-Clark, T. E., Kiros, F., Carr, M. J. and McAlexander, M. A. (2009). Transient receptor potential ankyrin 1 mediates toluene diisocyanate-evoked respiratory irritation. Am J Respir Cell Mol Biol 40, 756-762. doi:10.1165/rcmb.2008-0292OC
Taylor-Clark, T. E. and Undem, B. J. (2010). Ozone activates airway nerves via the selective stimulation of TRPA1 ion channels. J Physiol 588, 423-433. doi:10.1113/jphysiol.2009. 183301

Trevisani, M., Siemens, J., Materazzi, S. et al. (2007). 4-Hydroxynonenal, an endogenous aldehyde, causes pain and neurogenic inflammation through activation of the irritant receptor TRPA1. Proc Natl Acad Sci U S A 104, 13519-13524. doi:10.1073/pnas.0705923104

Vinken, M., Landesmann, B., Goumenou, M. et al. (2013). Development of an adverse outcome pathway from drug-mediated bile salt export pump inhibition to cholestatic liver injury. Toxicol Sci 136, 97-106. doi:10.1093/toxsci/kft177

Villeneuve D. L., Crump D., Garcia-Reyero N. et al. (2014). Adverse outcome pathway (AOP) development I: Strategies and principles. Toxicol Sci 2, 312-20. doi:10.1093/toxsci/kfu199

Xanthos, D. N. and Sandkuhler, J. (2014). Neurogenic neuroinflammation: Inflammatory CNS reactions in response to neuronal activity. Nat Rev Neurosci 15, 43-53. doi:10.1038/ nrn3617

\section{Conflict of interest}

The authors declare that they have no conflicts of interest.

\section{Acknowledgments}

The NIEHS Intramural Research Program supported, in part, this study. 